\title{
Analysis and Design of a Speed Controller for Switched Reluctance Motor Drive
}

\author{
Manuel Pereira ${ }^{1}$, Rui Esteves Araújo² \\ ${ }^{1}$ Doctoral Program in Electrical and Computer Engineering, Faculty of Engineering, University \\ of Porto, Porto, Portugal (ee12314@fe.up.pt) ORCID 0000-0002-6734-257X; ${ }^{2}$ INESC TEC, \\ Department of Electrical and Computer Engineering, Faculty of Engineering, University of \\ Porto, Porto, Portugal (raraujo@fe.up.pt) ORCID 0000-0002-7866-9068
}

\begin{abstract}
This paper presents a speed control of the reluctance machine for electric drive applications with fast dynamic demand. To get high-performance speed control, a cascade control algorithm is developed based on linear control technique. The controller is designed using the Root Locus Methodology and implemented on a numerical simulation platform. The design using Root Locus Methodology proved to be a viable approach and showed that various problems associated with the structural torque ripple of the electric motor can be solved. An important aspect of this work is the role played by model linearization in testing the sensitivity of the controller performance to specific parameter changes. The controller is applied to a simulated non-linear switched reluctance motor model in order to evaluate their performances. Simulation results showed that high-performance control for Switched Reluctance Motor has been achieved.
\end{abstract}

Author Keywords. Switched Reluctance Motor, Reluctance Motor Drives, Linear Control, Computer Aided Design.

Type: Research Article

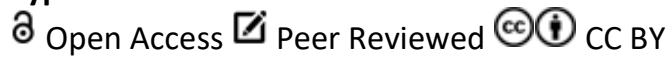

\section{Introduction}

In last decades, Switched Reluctance Motor (SRM) has been gaining much attention both in scientific and industrial communities due to its great advantages, such as fault tolerance and low cost, among other factors (Boldea and Nasar 2006). In recent years there has been a substantial increase of studies and reviews on SRM. For example, in Bostanci et al. (2017), a comparative study over the most common electrical machines with a focus on SRM is done, where some performance indicators such as power density, efficiency, torque ripple, vibration, noise and fault tolerance are compared. Another good example could be found in Gan et al. (2018) where is made a complete review and classification of advanced technologies, both in SRM topologies and in control. The methods, advantages and limitations of these technologies are summarized and compared. It is concluded that improving the SRM control strategy is a more cost-effective way to deal with the SRM noise and torque ripple. The main aspects of machine modeling, design and control are very well presented in Burkhart et al. (2017).

The most appreciated advantage of this motor is the non-use of neither rare elements nor windings on the rotor. Other advantages are its power density, which can be similar to the induction motor, the simplicity and the low manufacturing cost when compared to traditional motors. Also, as control is independent by phase, the motor can be very robust to failures (Bostanci et al. 2017). These advantages make SRM a possible future leader motor in variable speed applications. On the other side, SRM main disadvantages result from its own operating 
principle. Strong saturation in the magnetic core during steady-state operation makes the motor control due to its non-linear torque-per-ampere characteristic. These drawbacks have led to search for complex nonlinear control methodologies to compensate the motor nonlinearity and torque ripples. Many efforts have been made in the past to address these challenges. Recently, some complex works on non-linear models and non-linear controllers were proposed, for example in Divandari, Rezaie, and Ranjbar (2018) and Fabianski (2017), but this solution imposes the use of microprocessors or signal processors (DSPs) having adequate computing capacities. An important aspect of their use is the cost of solution. Usually, the more powerful digital microcontrollers are used the more the cost of solution is increased, which could become prohibitively expensive for some applications.

Motivated by the aforementioned limitations, this paper addresses the identified limitations via linear cascade control design. First, a high-bandwidth current control is designed with internal model dynamics. Second, a speed controller is formulated and designed based on a linearized model of the SRM. The proposed speed control scheme targets the problem of fast dynamics with a necessary commutation strategy, which ensures the speed regulation and robustness in the presence of parameter mismatches or load disturbances. This paper will focus on the details for linearization of the mathematical model of the motor and on the method for tuning the controller parameters. The importance of the work done consists in having a simple and solid explanation of the approach to determine the linear controllers. Also, the sensitivity analysis for some parameters is carried out which is a plus point because clarify how the poles and zeros of system react under different circumstances.

The remaining sections of this paper are organized as follows; section 2 will briefly describe the mathematical model of SRM and its linearization. Section 3 discusses with detail the design and controller synthesis. Section 4 will discuss the simulations results. Finally, in section 5 are the conclusions and future research on the subject.

\section{Background}

\subsection{Switched reluctance motor}

Switched reluctance motor is constituted by a stator, with concentrated windings disposed around polar cores, and by a rotor, composed by salient poles free of windings or magnets. Normally, each stator phase is composed by a couple of windings diametrically opposed.

When a stator phase is magnetized it is generated a closed magnetic field between the stator, the air gap and the rotor. This magnetic field tends to minimize the reluctance by reducing the air gap which creates a rotor movement. When a stator pole is aligned with a rotor pole it is said to be in the position of minimum reluctance and when they are unaligned it is said to be in the position of maximum reluctance. This characteristic of the motor makes possible to create a rotational movement of the rotor by magnetizing and demagnetizing each phase in the right position of the rotor. This SRM principle is explained with detail in Boldea and Nasar (2006).

Although SRM has a simple construction, the magnetic core is subjected to saturation making this a non-linear machine. For instance, the mechanism of torque generation is nonlinear and consequently it is not so simple to implement the speed regulation of this machine as can be seen in Husain (2002). The nonlinear magnetic characteristics associated with motor happen because of saturation and variable air gap which cause the magnetic flux linkage a nonlinear function of stator current and rotor position. Figure 1 shows the magnetization characteristic of an SRM machine, which will be studied in this work. 


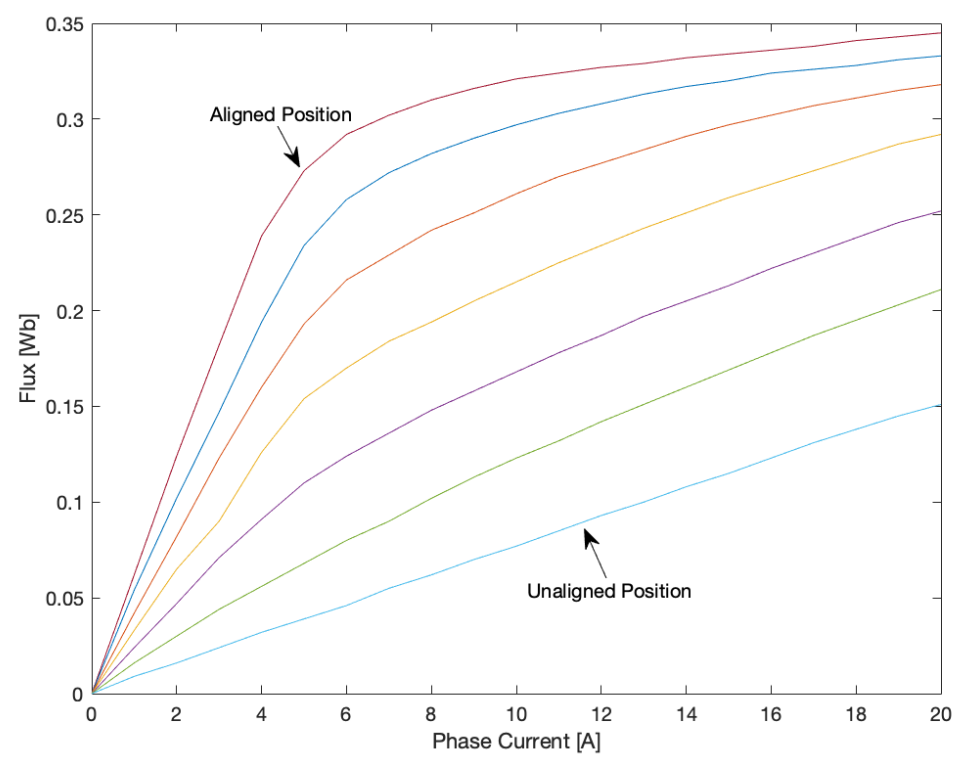

Figure 1: Flux-linkage curves of SRM

\subsection{Model equations}

In this section, the mathematical model of SRM is briefly presented in the following lines. Since this is an electromechanical energy converter, his model is described by four equations:

- Voltage equation

$$
V=R_{s} . i+\frac{d \Psi\left(i, \theta_{r}\right)}{d t}
$$

where $V, R_{S}, i$ and $\Psi\left(i, \theta_{r}\right)$ are, respectively, the phase voltage, the stator resistance, current and flux linkage, for each phase;

- Magnetic equation

$$
\Psi=L\left(i, \theta_{r}\right) . i
$$

where $L\left(i, \theta_{r}\right)$ is the phase self-inductance;

- Electromechanical equation

$$
\begin{gathered}
T(i)=\left(\frac{\delta W_{c}\left(\theta_{r}\right)}{\delta \theta_{r}}\right)_{i \text { constant }} \\
W_{c}=\int_{0}^{i} \Psi\left(i, \theta_{r}\right) d i
\end{gathered}
$$

where $W_{c}$ is the co-energy;

- Mechanical equation

$$
T-T_{L}=J \frac{d \omega_{r}}{d t}+B \cdot \omega_{r}
$$

where $T, T_{L}, J, B$ and $\omega_{r}$ are, respectively, the instantaneous torque, the mechanical load, the machine inertia, the viscous friction coefficient and the rotor angular speed.

The non-linearity of the SRM lies essentially in the magnetic equation since it represents the magnetic curves (see Figure 1). Due to this, it is in the magnetic equation that lies the complexity of SRM modeling. Further details are referred in Ahn (2011).

\subsection{Model simplification and linearization}

Even under simplifying assumptions such as perfect symmetry of stator windings, perfect symmetry of the magnetic circuit (zero mutual flux linkage in the motor phases), absence of hysteresis, the model of SRM in machine variables are quite involved due to the nonlinear dependency of phase self-inductance on the variable rotor position. The assumptions of nonsaturating magnetic structure and steady-state in the phase current are commonly performed 
in order to obtain an equivalent self-inductance that only depends on the rotor position, $L\left(i, \theta_{r}\right) \rightarrow L\left(\theta_{r}\right)$. With this simplification, the instantaneous torque can be easily calculated by

$$
T=\frac{d\left(\int_{0}^{i} L\left(\theta_{r}\right) \cdot i d i\right)}{d \theta_{r}}=\frac{i^{2}}{2} \frac{d L\left(\theta_{r}\right)}{d \theta_{r}}
$$

Before proceeding to the final equations, note that the torque is a function of $\frac{d L}{d \theta^{\prime}}$, the derivative of the phase inductance with respect to angular position of rotor.

Substituting the expression 6 for the instantaneous torque per phase into Equation 5 and bringing together the voltage Equation 1 and flux-current Equation 2, the complete set of electric motor equations is obtained:

$$
\begin{gathered}
\frac{d i}{d t}=-\frac{R_{S} \cdot i}{L(\theta)}-\frac{1}{L(\theta)} \frac{d L(\theta)}{d \theta} \omega_{r} . i+\frac{V}{L(\theta)} \\
\frac{d \omega_{r}}{d t}=\frac{1}{2 J} i^{2} \frac{d L(\theta)}{d \theta}-\frac{B}{J} \omega_{r}-\frac{T_{L}}{J}
\end{gathered}
$$

It is clear from Equations 7 and 8 that there are terms that change continuously throughout the rotor position and hence showing a great deal of non-linearity of the model. Therefore, in order to ensure a model more tractable, one must get rid of the dependency of rotor position. Towards this end, constant parameters are defined in the next lines in order to make another common approximation (see Equations 15-17). The effect of rotor position on the selfinductance and its derivative with respect to angular position of rotor had been neglected, which are calculated to be constants. In particular, the mean and the derivative of the highest and lowest points, of self-inductance, are used, respectively.

Even so, the SRM model remains highly non-linear, which is not suitable for the synthesis linear controllers. The simplest solution to this is to linearize the motor equations around a desired operating point and then apply linear control techniques. Even though the validity of this approach has some limitations, this solution is frequently applied in practice.

The first step in linearizing the equations system is to approximate the variables as:

$$
\begin{gathered}
i=i_{o}+\delta i \\
\omega_{r}=\omega_{r o}+\delta \omega_{r} \\
V=V_{o}+\delta V \\
T_{L}=T_{L o}+\delta T_{L}
\end{gathered}
$$

Where the subscript $o$ is used to define the variable value at the steady-state operation point and the $\delta$ represents incremental deviations of the variable in the vicinity this point. Substituting Equations 9-12 into Equations 7 and 8 and after some simplifications and manipulations the new equations are obtained, in time and frequency domain by Laplace transform $(\mathcal{L})$ :

$$
\begin{gathered}
\frac{d \delta i}{d t}=-\frac{R_{e q}}{L} \delta i-\frac{\delta_{e}}{L}+\frac{\delta V}{L} \rightarrow \mathcal{L} \rightarrow \delta I(s)=\frac{\delta V(s)-\delta_{e}}{L s+R_{e q}} \\
\frac{d \delta \omega_{r}}{d t}=\frac{K_{b}}{J} \delta i-\frac{B}{J} \delta \omega_{r}-\frac{\delta T_{L}}{J} \rightarrow \mathcal{L} \rightarrow \delta \omega_{r}(s)=\frac{K_{b} . \delta I(s)-\delta T_{L}(s)}{J s+B}
\end{gathered}
$$

With the constants, such as:

$$
\begin{gathered}
R_{e q}=R_{s}+\frac{d L}{d \theta} \omega_{r o} \\
K_{b}=\frac{d L}{d \theta} i_{o}
\end{gathered}
$$




$$
\delta_{e}=\frac{d L}{d \theta} i_{o} \cdot \delta \omega_{r}
$$

Where $R_{e q}, K_{b}$ and $\delta_{e}$ represent, respectively, the equivalent resistance, the torque current gain and the induced back-electromotive force. For further details on simplification of the model, the reader is suggested to consult Krishnan (2001).

Now that the motor has been linearized, a linear control technique can be applied to it. $A$ conventional block diagram of the SRM in the frequency domain is shown in Figure 2, which represents the whole system. The system inputs are the variations of voltage and load. The variation of angular velocity is the output of the system.

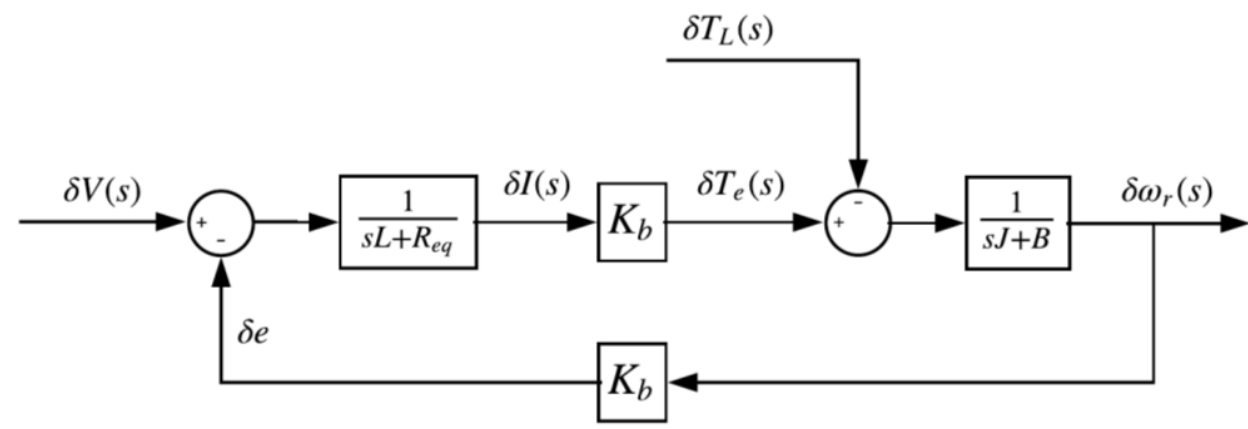

Figure 2: Linearized SRM model

\section{Control System}

The goal of this section is to present a concise, but relatively complete, synthesis of linear controllers for the model in Figure 2. As usually, in motor drives, current and velocity are controlled in a cascade speed control configuration. The same control configuration is used in this work. In fact, from the diagram present in Figure 2 the torque variation is proportional to the current variation. Thus, it would be conveniente if the current was the manipulation variable since one could easily define the motor torque by impose the current. This way a cascate structure is employed where the current control loop is subordinated to the speed controller. In other words, the voltage is forced by a current controller to go to whatever value necessary to obtain the desired current.

For this study, the parameters of a well-described SRM presented in Soares and Branco (2001) are used. The motor has three-phases constituted by six stator poles and four rotor poles. The complete list of parameters is repeated in Appendix A for convenience of the reader.

The controllers must be designed according to the application the motor may have and according to the motor specifications. It should be pointed that the synthesis principle for cascade control, one must first perform the tuning of the inner loop, i.e. the current control loop. The following simplifying assumptions are made: - the back electromotive force induced (Equation 17) is taken to be a disturbance which, from the point of view of the inner loop changes very slowly as compared with the dynamic of current; - the power converter is approximated by a simple gain; - The motor operates in steady-state with constant load torque and the chosen operating point is $15 \mathrm{~A}, 165 \mathrm{rad} / \mathrm{s}$ and 5,8 N.m of produced electric torque.

\subsection{Current loop}

In the designing of the current controller, the transfer function $\frac{\delta I(s)}{\delta V(s)}$ is easily found using the block diagram reduction method, this way the transfer function appears as

$$
\frac{\delta I(s)}{\delta V(s)}=\frac{B+s J}{s^{2}(L J)+s\left(R_{e q} J+L B\right)+\left(R_{e q} B+k_{b}^{2}\right)}
$$


For the given parameters, the transfer function has a zero in $s=-14$ seconds $s^{-1}$ and poles in $s=-$ 324 seconds $^{-1}$ and $s=-36$ seconds $^{-1}$. As all poles have negative real part the system is stable. Is noted that the pole in $s=-324$ seconds $^{-1}$ is too far on the left when compared to the pole in $s=-$ 36 seconds $^{-1}$ and the zero in $s=-14$ seconds $^{-1}$. In this case the first pole corresponds to the current dynamics and the others pole and zero correspond to the mechanical dynamics.

Under these conditions the current controller can be represented as in Figure 3, where $C_{I}(s)$ is a $\mathrm{PI}$ compensator.

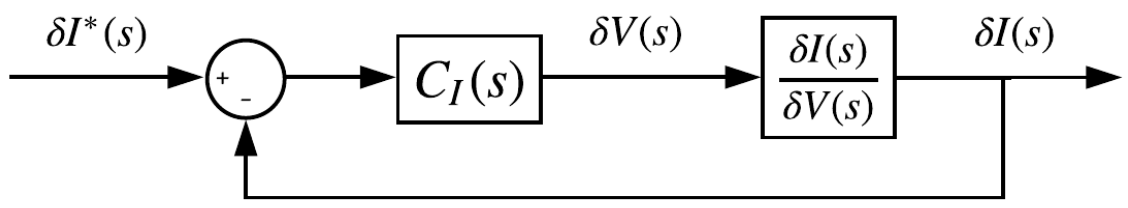

Figure 3: Current closed-loop diagram

PI controller consists of assign a pole in the origin, a zero where most desired and in choosing a gain. If the zero is put on the left side of the faster dynamic pole, this case in position $s=-550$ seconds ${ }^{-1}$, it is obtained a root locus for the controller gain where appears a circumference form, as shown in Figure 4. This way is possible to choose a gain where the system poles have symmetric imaginary part, and if the system gain changes, the system dynamic changes too, till a certain point where the poles leave the circumference form. The chosen gain, of current controller, is 20 , and the time constant of $1.8 \mathrm{~ms}$ giving a PI controller in the form $C_{I}(s)=$ $20\left(1+\frac{550}{s}\right)$.

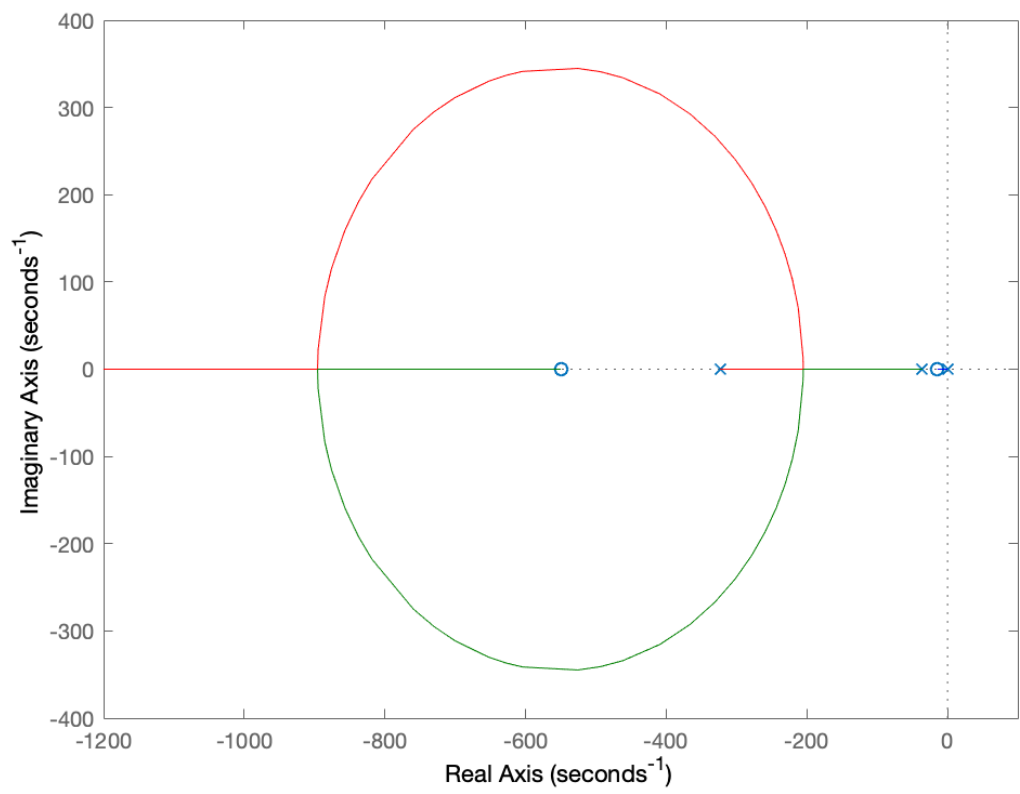

Figure 4: Root locus for current loop controller

\subsection{Speed loop}

Speed closed loop in Laplace domain is shown in Figure 5. The diagram presents two inputs, the variation of the reference speed and the variation of the load torque. The controller is designed as a servo problem (tracking an input reference). This way the parameters of PI controller are chosen to achieve two objectives: 1) tracking - if the reference speed is as unitstep function, then is required that the output must track the input with a settling time inferior to $200 \mathrm{~ms}$; 2) disturbance rejection - when the setpoint of speed is constant, the output response to step change of load torque must go to zero as $t \rightarrow \infty$. In section 3.3 the response 
of the system is observed, already with the designed controller, for a change in reference speed and for a change in load, separately.

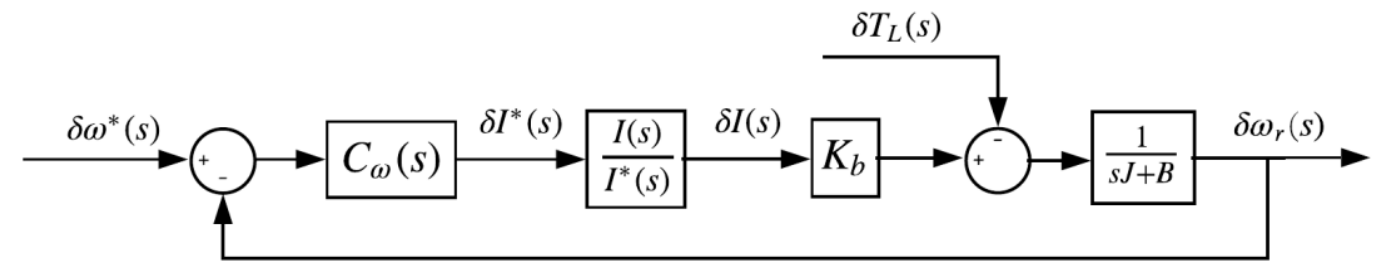

Figure 5: Speed closed-loop diagram

Speed controller must have a dynamic slightly faster than the mechanic dynamic and much slower than the current dynamic. These assumptions will allow the speed controller to be fast enough for the mechanical changes and slow enough to be decoupled from current control dynamics.

In Figure 6 the root locus for the closed-loop speed with a PI controller is shown. Same way as in the current loop, the PI was chosen to have a zero on the left side of the pole that represents the mechanical dynamic creating a circumference form in the figure as shown in the zoomed part. On the left side of the figure there are two poles and one zero which correspond to the current dynamic and the current controller. The chosen gain, of the speed controller, is 0.22 , and the time constant of $0.18 \mathrm{~s}$ giving a PI controller in the form $C_{I}(s)=0.22\left(1+\frac{5.5}{s}\right)$.

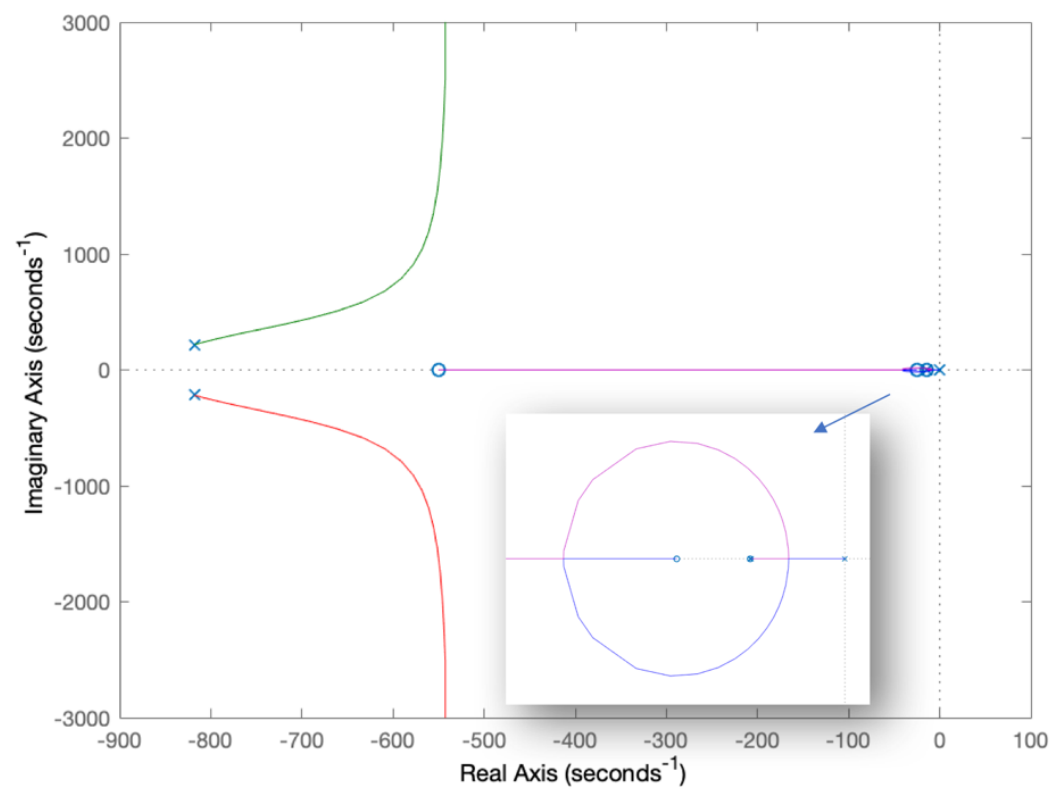

Figure 6: Root locus for speed loop controller

\subsection{Step response analysis}

With all control parameters calculated, selected transient responses to step changes of speed reference and load torque are shown in Figure 7 for different values of inductance (L). The point used to create the controllers is taken as a reference point and it is compared with other specific conditions. For further analysis of results, the closed-loop transfer functions for speed variation and load variation, we have:

$$
\begin{gathered}
\frac{\delta \omega(s)}{\delta \omega^{*}(s)}=\frac{C_{\omega}(s) \cdot \frac{\delta I(s)}{\delta I^{*}(s)} \cdot K_{b}}{s J+B+C_{\omega}(s) \cdot \frac{\delta I(s)}{\delta I^{*}(s)} \cdot K_{b}} \\
\frac{\delta \omega(s)}{\delta T_{L}(s)}=-\frac{1}{s J+B+C_{\omega}(s) \cdot \frac{\delta I(s)}{\delta I^{*}(s)^{\prime}} \cdot K_{b}}
\end{gathered}
$$


The first set of simulations addresses the maximum inductance impact over the system response. One should keep in mind the current influence over this parameter, due to core saturation. Temperature may have some impact too. This parameter highly changes, nonlinearly, with the current and may also change with temperature. Figure 7 shows the response of the designed system comparing the maximum self-inductance value for plus twenty percent and minus twenty percent of the tabled value.
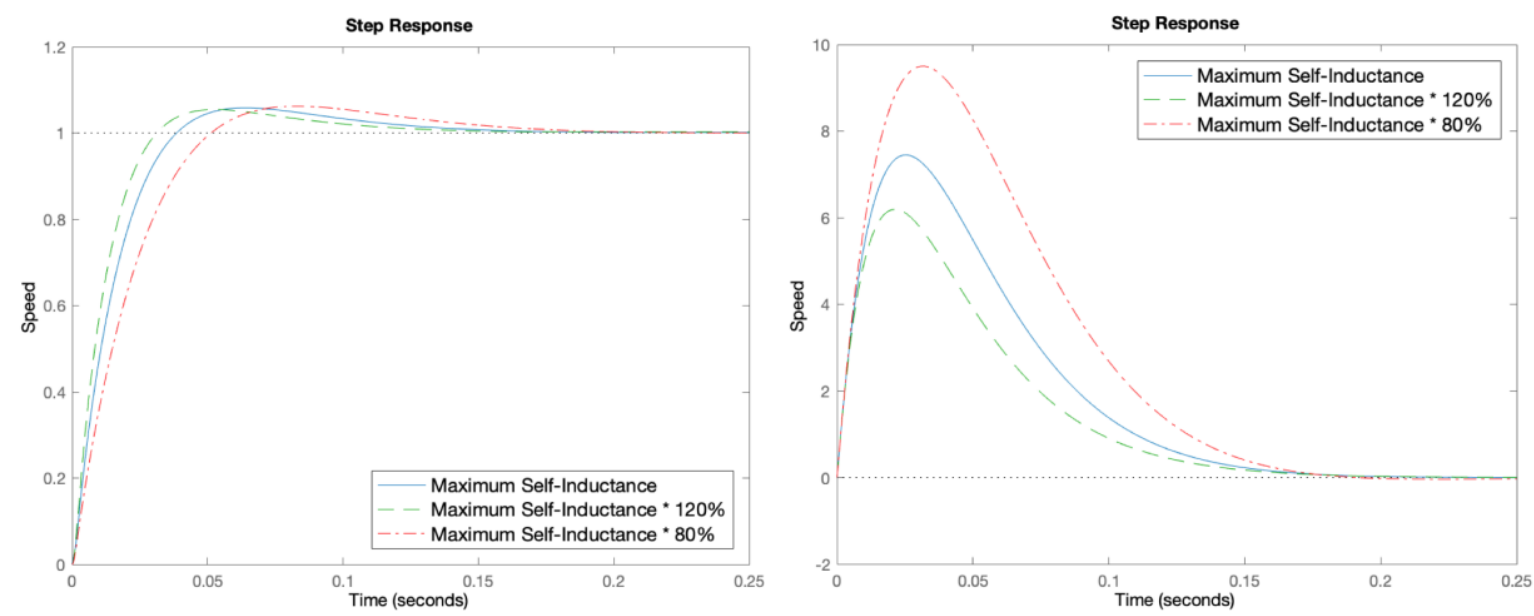

Figure 7: Speed transients under step change of speed reference for maximum inductance variation (left side), and speed transients under step change of load torque (right side)

The left figure shows the result of a speed step and no-load variation, and the right figure a load step and no speed variation. Note that taking account of the effect of inductance changes only the character of the dynamic curves, the steady-state values are the same. By numeric results, as shown in Table 1 , in increasing the maximum inductance value, the system dynamics is increased. Looking for transfer functions and considering current loop to have no dynamic, for being much faster, only $K_{b}$ changes with the maximum inductance change. In this case, increasing $K_{b}$ puts the poles and zeros more on the left plane, increasing the system dynamics.

\begin{tabular}{|c|cc|cc|}
\cline { 2 - 5 } \multicolumn{1}{c|}{} & \multicolumn{2}{c|}{ Speed Step } & \multicolumn{2}{c|}{ Load Step } \\
\cline { 2 - 5 } \multicolumn{1}{c|}{} & Settling Time (2\%) & Overshoot & Settling Time (2\%) & Peak \\
\hline Reference & 0.119 & 5.825 & 0.160 & 7.449 \\
Reference * 120\% & 0.102 & 5.434 & 0.159 & 6.193 \\
Reference * 80\% & 0.147 & 6.213 & 0.164 & 9.501 \\
\hline
\end{tabular}

Table 1: Numeric results of step response for maximum inductance variation

Another set of simulations addresses the fact the motor does not operate always in the same operating point. Two other operating points are used: Operating Point 1 with a steady-state current and speed of $10 \mathrm{~A}$ and $35 \mathrm{rad} / \mathrm{s}$, respectively, and Operating Point 2 of $17 \mathrm{~A}, 200 \mathrm{rad} / \mathrm{s}$, respectively. These new operating points represent the system operating at low and high speeds with a current that allows having a significant load. The step response for the three operating points is shown in Figure 8 and the numeric results are in Table 2. 

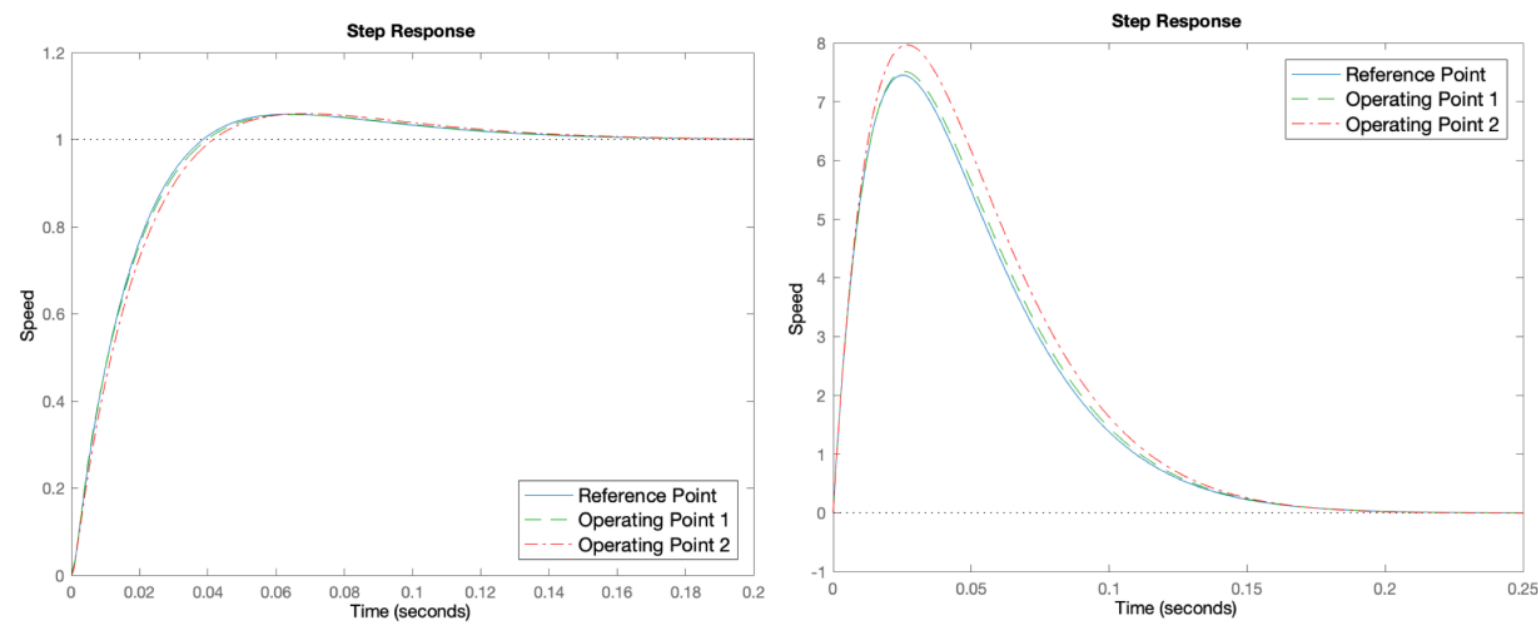

Figure 8: Step response for different operating points by speed perturbation (left side), and load perturbation (right side)

By the figures and by the numeric results, there are no significant differences in the system dynamics. Looking for the transfer functions and considering again that the current loop has no dynamic, it is seen that steady-state speed has no effect in the system. Also, as values $J$ and $B$ are kept the same, only $K_{b}$ changes for different points. Reminding that $K_{b}$ is the product of the inductance derivative with the steady-state current. In the model of Soares and Branco (2001), the calculation of $K_{b}$ for the range of $8 \mathrm{~A}$ to $20 \mathrm{~A}$, only varies from 0.3 to 0.4 units, which is a small difference, not having much effect on the system.

\begin{tabular}{|c|cc|cc|}
\cline { 2 - 5 } \multicolumn{1}{c|}{} & \multicolumn{2}{c|}{ Speed Step } & \multicolumn{2}{c|}{ Load Step } \\
\cline { 2 - 5 } & Settling Time (2\%) & Overshoot & Settling Time (2\%) & Peak \\
\hline Reference & 0.119 & 5.825 & 0.160 & 7.445 \\
Operating Point 1 & 0.121 & 5.734 & 0.161 & 7.511 \\
Operating Point 2 & 0.126 & 5.965 & 0.160 & 7.965 \\
\hline
\end{tabular}

Table 2: Numeric results of step response for different operating points

In conclusion, the system, with the designed controllers, has a good performance not only for the reference point but also for different scenarios. Changing the maximum inductance parameter has revealed to be more significant than changing the operating point for different speeds and currents, with the simulated parameters.

\section{Results}

In the previous section, a linearization of a simplified SRM model was made, which was the basis for the control design and small signal analysis studies. In this section, a simulation environment based on Simulink ${ }^{\circledR} /$ Matlab $^{\circledR}$ is developed. The designed control system is tested with a non-linear SRM model. Used parameters for SRM and controllers are also presented in Appendix A.

\subsection{Simulation environment}

Figure 9 shows the Simulink model of the SRM drive. As it can be seen, its structure derives from the schemes depicted in Figures 3 and 5. The motor model was integrated in a block SRM. The structure of power converter is in half-bridge. As the motor has three phases, the converter has six transistors and six diodes. This converter has the advantages of having only positive currents and is able to regenerate the non-used energy in the motor to the DC voltage source. 


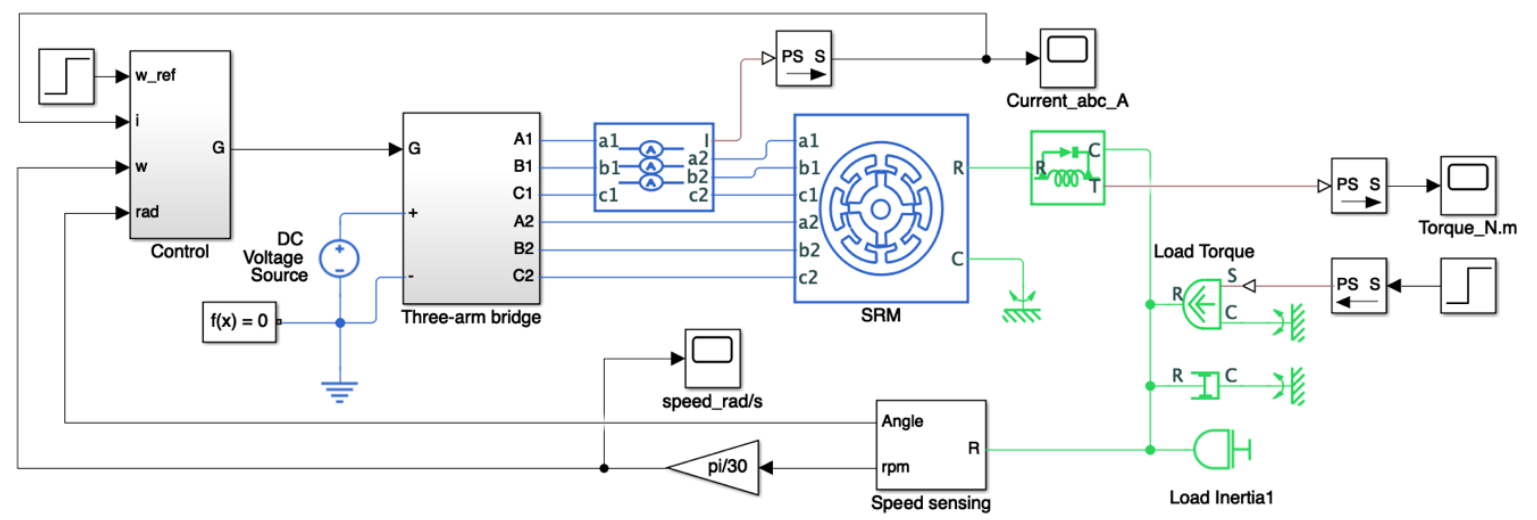

Figure 9: Block diagram of simulated non-linear SRM

The motor control is constituted by a speed PI controller, a current PI controller, a power converter and a block that implement the instant of phase triggering. Both $\mathrm{PI}$ controller gains were calculated in sections 3.1 and 3.2. A Pulse Width Modulation (PWM) block is used to converter the current controller output into a series of logical signals that define the state of the power converter switches. The block which triggers the phase opening and closing has the function to compare the rotor position with pre-defined values, in order to impose whether each phase must conduct or not. This block interacts with the current reference, generated by the speed controller. For the simulations, a current conducting range between 0 and 40 degrees is used, where in the range of 0 to 45 degrees is produced torque in positive direction. The study examines system behavior for speed tracking and load perturbation. In particular, the dynamic characteristics under step changes of speed reference and load torque.

\subsection{Simulation results}

A first approach is to check if the chosen points can be obtained in the simulations. Figure 10 and Table 3 show the steady-state results for the three operation points considered in the previous section, Reference Point, Operating Point 1 and Operating Point 2.

\section{Reference Point $\quad$ Operating Point $1 \quad$ Operating Point 2}
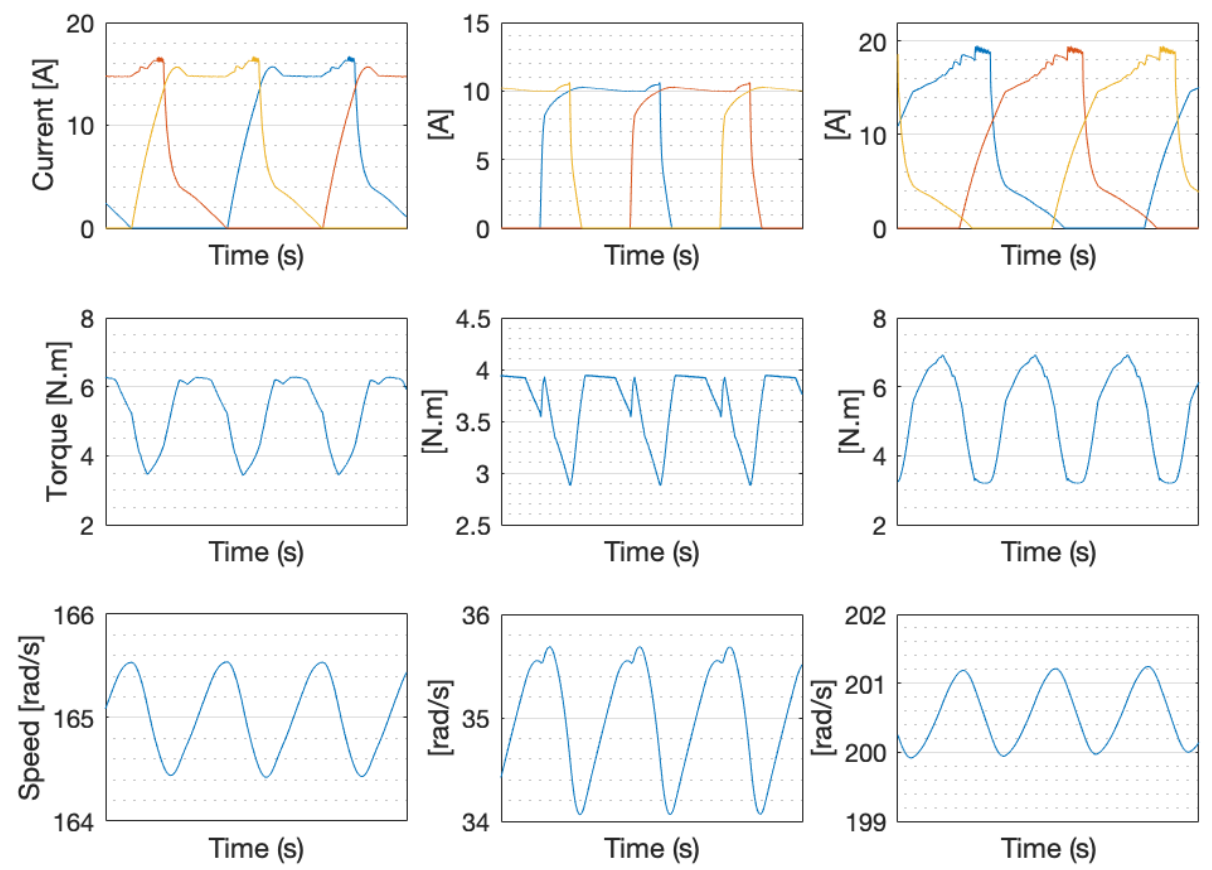

Figure 10: Simulation for different operating points 


\begin{tabular}{|c|ccc|}
\cline { 2 - 4 } \multicolumn{1}{c|}{} & Reference & Operating Point 1 & Operating Point 2 \\
\hline Mean Torque [N.m] & 5.22 & 3.63 & 5.16 \\
Mean Speed [rad/s] & 165.04 & 34.99 & 200.61 \\
Load Torque [N.m] & 2.2 & 3.0 & 1.5 \\
\hline
\end{tabular}

Table 3: Numeric results for different operating points

Ideally, the current waveform would be a square wave, almost as what happens in Operating Point 1. But as speed increases so does the back-emf, making the current dynamic much slower. In Operating Point 1 it is easily seen that the current has a steady-state value around $10 \mathrm{~A}$, as expected. Reference Point looks almost as a trapezoidal waveform but the steadystate value is around the expected $15 \mathrm{~A}$. At high speed, Operating Point 2, the back-emf makes it impossible to keep a steady-state value where the current has a slow dynamic.

Speed and torque results, due to the characteristic of the motor, presents a significant ripple component. In spite of that, the numeric mean values correspond to the desired values.

Due to the back-emf and by the model's limitation of the maximum current, which is $20 \mathrm{~A}$, the results of transient response for the linear model cannot be compared with the ones in the non-linear model.

In Figure 11, a speed transient is shown when the motor is operating at Reference Point.

Speed set point goes from $165 \mathrm{rad} / \mathrm{s}$ to $175 \mathrm{rad} / \mathrm{s}$ at $0.35 \mathrm{~s}$, and the load torque is kept constant with a value of $-2.2 \mathrm{~N}$.m. By Figure 11, the current controller acts instantaneously at the moment of change. The speed grows till $175 \mathrm{rad} / \mathrm{s}$ under $0.05 \mathrm{~s}$ and the final values correspond to the imposed reference.
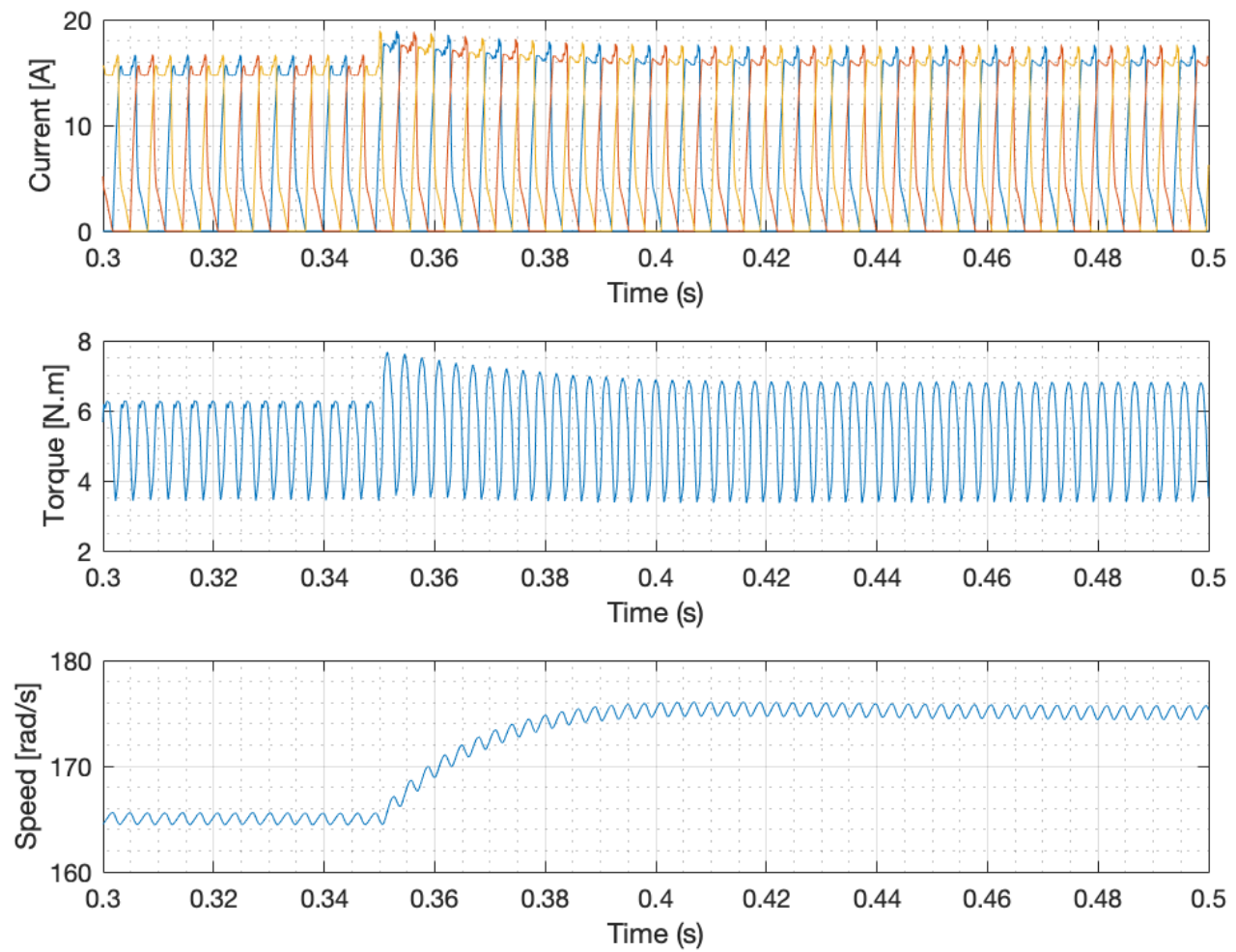

Figure 11: System responses to a steep change of speed reference signal 

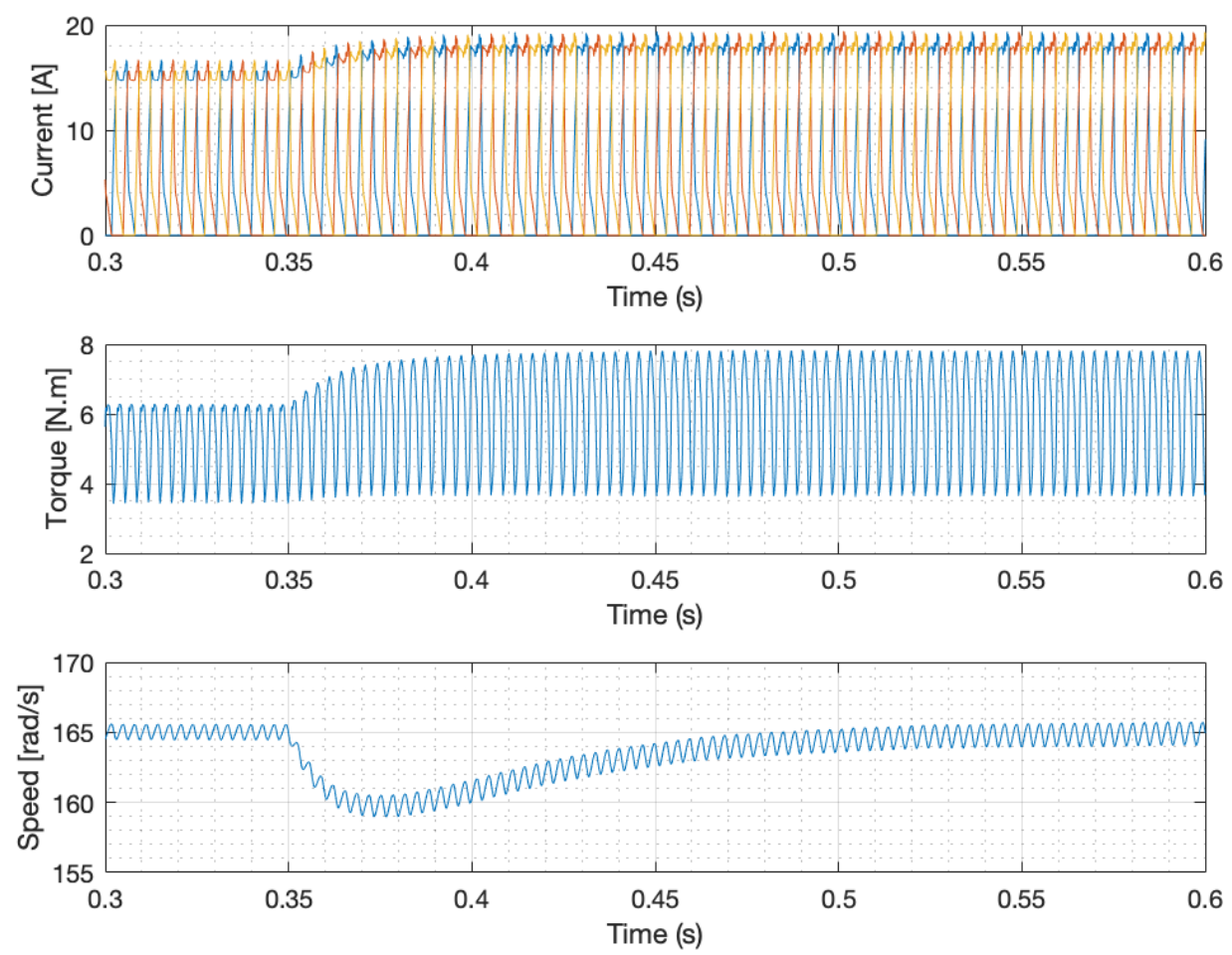

Figure 12: Motor transients under step change of load torque

In the next simulation, the control system is affected by an unknown step change of load torque perturbation when the reference speed is $165 \mathrm{rad} / \mathrm{s}$. The unknown perturbation load torque is applied at $0.35 \mathrm{~s}$. The responses of current, instantaneous torque and rotor speed are shown in Figure 12. The load torque value is increased from -2.2 N.m to -3.0 N.m.

The good dynamic performance of control system is evident in Figure 12. It is possible to see that the control system is able to compensate the torque perturbation with a slight increase of the current (and instantaneous torque). The speed decay to $159 \mathrm{rad} / \mathrm{s}$ and under $0.2 \mathrm{~s}$ the system has recovered the reference speed.

\section{Conclusions}

This paper presents a control method for SRM, taking into account its non-linear features. Starting from a non-linear SRM model, the model is based on its linearization and simplification. Furthermore, a speed control system for a SRM based on cascade PI controllers was designed and implemented. Although the linearization is a very simplified case of the model, it was possible to have high-performance results in the non-linear simulations. Results for speed tracking and load perturbation showed a fast and coherent response of the designed controllers. A speed perturbation of $10 \mathrm{rad} / \mathrm{s}$ was recovered in less than $0.05 \mathrm{~s}$, and a load perturbation of $0.8 \mathrm{~N} . \mathrm{m}$ was recovered in less than $0.2 \mathrm{~s}$. These results demonstrate the speed regulation and robustness of the method used. It is proved that despite the nonlinearities of the motor it is possible to design the controllers based on linear model resulting in control system with good performance in low and high-speed range of operation.

In a future work, we plan to develop a method to enhance the current loop and to decrease the ripple component, it would be interesting to improve the developed controller with the use of sharing torque functions. This idea is essentially attached to the instants of phase triggering and not directly with the linear controllers. 


\section{References}

Ahn, JIn-Woo. 2011. "Switched reluctance motor". In Torque control, edited by Moulay Tahar Lamchich, 201-52. InTechOpen. https://doi.org/10.5772/10520.

Boldea, Ion, and Syed A. Nasar. 2006. Electric drives. Boca Raton: CRC Press.

Bostanci, Emine, Mehdi Moallem, Amir Parsapour, and Babak Fahimi. 2017. "Opportunities and challenges of switched reluctance motor drives for electric propulsion: A comparative study". IEEE Transactions on Transportation Electrification 3, no. 1 (january): 58-75. https://doi.org/10.1109/TTE.2017.2649883.

Burkhart, Bernhard, Annegret Klein-Hessling, lliya Ralev, Claude P. Weiss, and Rik W. De Doncker. 2017. "Technology, research and applications of switched reluctance drives". CPSS Transactions on Power Electronics and Applications 2, no. 1: 12-27. https://doi.org/10.24295/CPSSTPEA.2017.00003.

Divandari, M., B. Rezaie, and A. N. Ranjbar. 2018. "Improved analytical nonlinear model for Switched Reluctance Motor using Gaussian distribution probability density function". Iranian Journal of Science and Technology - Transactions of Electrical Engineering 42, no. 3 (september): 343-56. https://doi.org/10.1007/s40998-018-0079-z.

Fabianski, Bogdan. 2017. "Optimal control of switched reluctance motor drive with use of simplified, nonlinear reference model". In Proceedings of the 2016 17th International Conference on Mechatronics - Mechatronika, ME 2016, 1-8. IEEE. https://ieeexplore.ieee.org/document/7827812.

Gan, Chun, Jianhua Wu, Qingguo Sun, Wubin Kong, Hongyu Li, and Yihua Hu. 2018. "A review on machine topologies and control techniques for low-noise switched reluctance motors in electric vehicle applications". IEEE Access 6, (may): 31430-43.

https://doi.org/10.1109/ACCESS.2018.2837111.

Husain, Iqbal. 2002. "Switched reluctance machines". In The power electronics handbook edited by Timothy L. Skvarenina, 13-1:13-19. Boca Raton, Florida: CRC Press. https://doi.org/10.1201/9781420037067.

Krishnan, R. 2001. Switched reluctance motor drives: Modeling, simulation, analysis, design, and applications. Industrial Electronics Series. Boca Raton: CRC Press. https://doi.org/10.1201/9781420041644.

Soares, F., and P. J. Costa Branco. 2001. "Simulation of a 6/4 switched reluctance motor based on Matlab/Simulink environment". IEEE Transactions on Aerospace and Electronic Systems 37, no. 3 (july): 989-1009. https://doi.org/10.1109/7.953252.

\section{Appendix}

\begin{tabular}{cc}
\hline \multicolumn{2}{c}{ SRM Parameters } \\
\hline Number of stator poles & 6 \\
Number of rotor poles & 4 \\
Voltage & $150 \mathrm{~V}$ \\
Stator Resistance & $1.3 \Omega$ \\
Inertial Value & $0.0013 \mathrm{~kg}^{*} \mathrm{~m}^{\wedge} 2$ \\
Friction Coefficient & $0.0183 \mathrm{~N}^{*} \mathrm{~m} /(\mathrm{rad} / \mathrm{s})$ \\
Minimum Inductance & $8 \mathrm{e}-3 \mathrm{H}$ \\
Maximum Inductance & $60 \mathrm{e}-3 \mathrm{H}$ \\
\hline
\end{tabular}

\begin{tabular}{|c|c|}
\hline \multicolumn{2}{|c|}{ PI Controllers Parameters } \\
\hline Current Kp & 20 \\
\hline Current $\mathrm{K}_{\mathrm{I}}$ & 550 \\
\hline Speed Kp & 0.22 \\
\hline Speed KI & 5.5 \\
\hline
\end{tabular}

Appendix A - Parameters of SRM (left side), and parameters of designed controllers (right side) 